\title{
Effects of Functional Shoes on Joint Moment, Ground Reaction Force, and EMG
}

\author{
Chong-hoon Lee \\ Department of Sports Science, Seoul National University of Science \& \\ Technology, Seoul, Korea \\ leejh36@snut.ac.kr
}

\begin{abstract}
Unstable shoes or high heel shoes may cause injury. From these results, it can be inferred that functionally superior shoes affect human walking positively. The purpose of this study was to examine biomechanical aspects of shoes that are being developed to have similar effect as if bare foot walking. This study examined the effects of functional shoes which is being developed to have similar effects of bare foot walking through subject test with quantitative analysis. Loading patterns during walking revealed with joint moments
\end{abstract}

Keywords: Shoes, EMG, Ground Reaction Force, Joint Moment

\section{Introduction}

During walking, shoe's primary function is to dissipate ground reaction force and lessens the magnitude of stress on joints such as knees or hip and eventually prevents injury. In modern years, a variety of materials, stiffness, and out-sole types of shoes are being tested in an effort to improve functionality of shoes. In 1930s, shoe development relied on participant's subjective opinion or rather non-scientific methods but in early 1970s Henning and colleagues started to use capacitive method which uses analog signal to measure pressure distribution and some researchers began to approach it with more scientific ways (Nigg \& Bahlsen, 1986). After those times, as modern industries developed, shoe industry developed as well in 1980s and 1990s and in recent years, numerous types of shoes are being developed. One of the important factors of shoes' function is the impact force at the heel contact period. During walking vertical ground reaction force from heel contact exceeds 2 to 3 times of body weight (Mann, 1980), and heel goes through, depending on surface and shoe types, 20 to 50 times more acceleration than from (Cavanagh et al., 1985). Therefore, it has been reported that in order to prevent injury from running, it is important to design shoes that minimize impact force from the ground. Our body takes force from the ground as the same amount of impact force during walking or running. The force moves up through kinetic chain starting from feet. Any defect in shoe's function will aggravate this and influence our body in a negative fashion. Normally shoes deform as time goes and tend to lose ability to absorb impact force (Kuk chang su, 1999).

Kerrigan, Lelas \& Karvosky (2001) found relationship between women's shoe-heel types and knee osteoarthritis. With 20 healthy women walking, they found a $30 \%$ increase in torques on knees with wider-heel shoes when compared to bare foot and $26 \%$ and $22 \%$ increases in knee valgus torques when they were wearing wider-heel and narrow-heel shoes, respectively. Previous studies indicate that types of shoes may affect impact force and joint moments on body. In other words, unstable shoes or high heel shoes may cause injury. From these results, it can be inferred that functionally superior shoes affect human walking positively. 
The purpose of this study was to examine biomechanical aspects of shoes that are being developed to have similar effect as if bare foot walking.

\section{Method}

\subsection{Subjects}

Ten asymptomatic healthy males without any previous injury that might have affected experimental protocols were recruited. Prior to participation, all subjects were briefly instructed protocols and signed an informed consent form.

\section{Table 1. Subject Characteristics (Mean \pm St.Dev.)}

\begin{tabular}{cccc}
\hline Subject & Height $(\mathrm{cm})$ & Mass $(\mathrm{kg})$ & Age $($ year $)$ \\
\hline \hline 20 males & & & \\
$(\mathrm{n}=10)$ & $175.50 \pm 6.02$ & $70.00 \pm 6.15$ & $23.40 \pm 1.90$ \\
\hline
\end{tabular}

\subsection{Equipment}

A motion analysis system with 12 cameras $(100 \mathrm{~Hz})$ and a force plate $(1000 \mathrm{~Hz})$ were used to obtain kinetics and kinematics. EMG was used to measure muscle activities $<$ Table 2 and Figure 1>.

Table 2. Equipment

\begin{tabular}{cccc}
\hline Equipment & Company & Quantity & Compare \\
\hline \hline Motion & Motion & & \\
Analysis & Analysis & 12EA & $100 \mathrm{HZ}$ \\
System & System & & \\
Force plate & AMTI & \multirow{2}{*}{ 1EA } & $100 \mathrm{HZ}$ \\
\hline
\end{tabular}

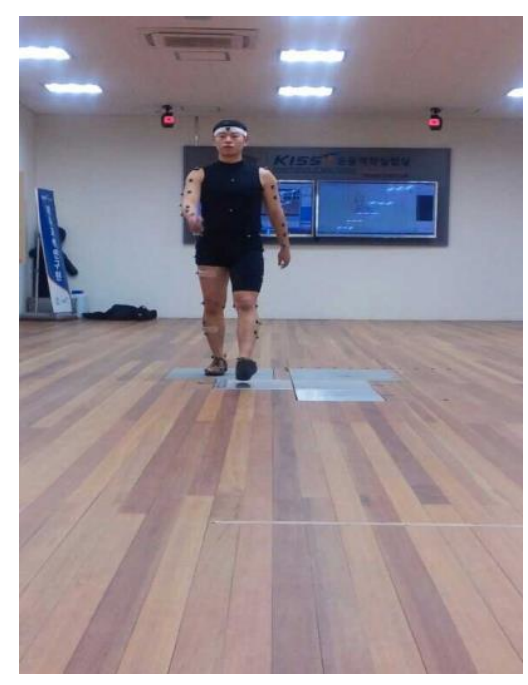

Figure 1. Attachment Placement of Land Mark

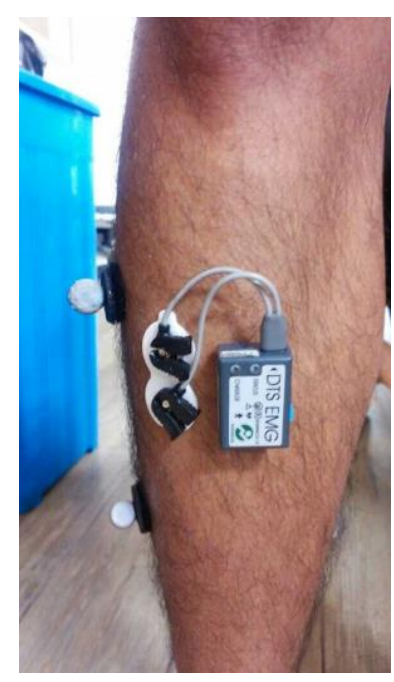

Figure 2. E.M.G 


\subsection{Experimental Protocol}

- Reflective marker placement (Figure 1)

- Preferred and natural walking speed

- $20 \mathrm{~m}$ walking distance

- Function shoes and bare foot walking

- Knee and ankle joint moments

- Ground reaction force

- EMG4. Data analysis

\subsection{Data Analysis}

Motion analysis system, force plate, and EMG data were exported and process with Motion Analyssis Track Manager, Visual3D, MyoReserach, Metlab, and Microsoft Execl (Figure 3).

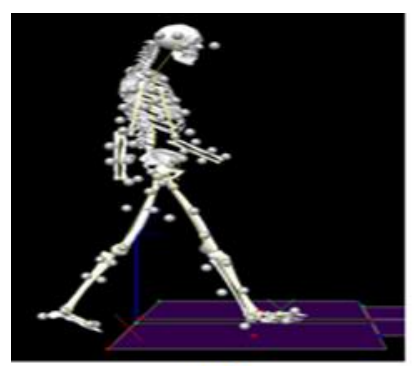

RHS

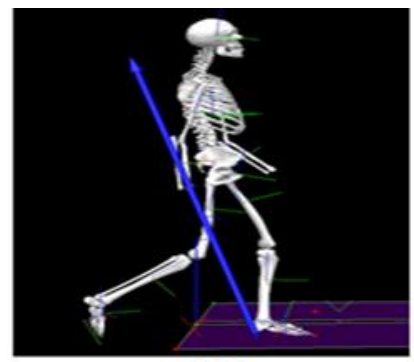

RMS

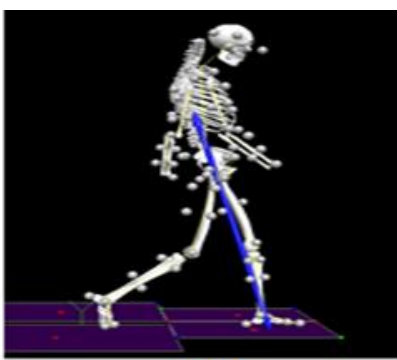

RTO

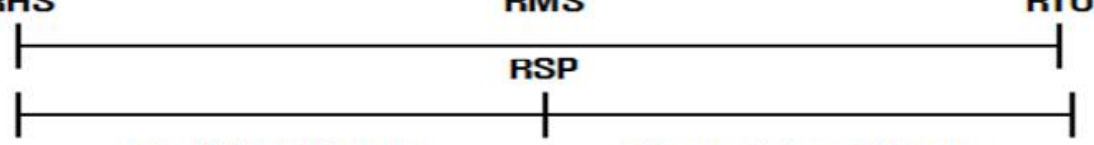

Braking Phase

Propulsive Phase

Figure 3. Event and Phase

\subsubsection{Event}

- Right heel strike (RHS): right heel contact

- Right mid stance (RMS): center of pressure above mid-foot

- Right toe take-off (RTO): right toe off

\subsubsection{Phase}

- Right Foot Supporting Phase (RSP): between RHS-RTO events, right foot stance

- Braking Phase (BP): between RHS-RMS events, loading response

- Propulsive Phase (PP): between RMS-RTO events, push off

\subsubsection{Dependent Variables}

- Ankle and knee joint moments

- Impact force: During BP phase, maximum anterioposterior, mediolateral, and vertical components of ground reaction forces

- Work amount: During PP phase, maximum anterioposterior, mediolateral, and vertical components of ground reaction forces

- EMG: iEMG from lower extremity muscles (Rectus femoris, Biceps femoris, Gastrocnemius, Tibialis anterior)

: Full wave rectification

: Band pass filter, 20 500 Hz 
: Integration

$$
\mathrm{IEMG}=\int_{t}^{t+T}|E M G(t)| \cdot d t
$$

\subsubsection{Statistical Analysis}

Multiple paired t-tests were performed using SPSS 21.0 and alpha level was set at $\mathrm{p}$ $<.05$.

\section{Results and Discussion}

\subsection{Ankle Moment}

Ankle moment about anteriorposterior axis occurs during pronation and supination. Significant differences were observed during BP in Maximum and minimum moments. It could be due to subject's impact force avoidance strategy when walking bare foot and may be also related to observed shorter stride length in this study. Ankle moments during other phases were similar between the conditions (Figure 4, 5).

Table 3. Ankle Moment

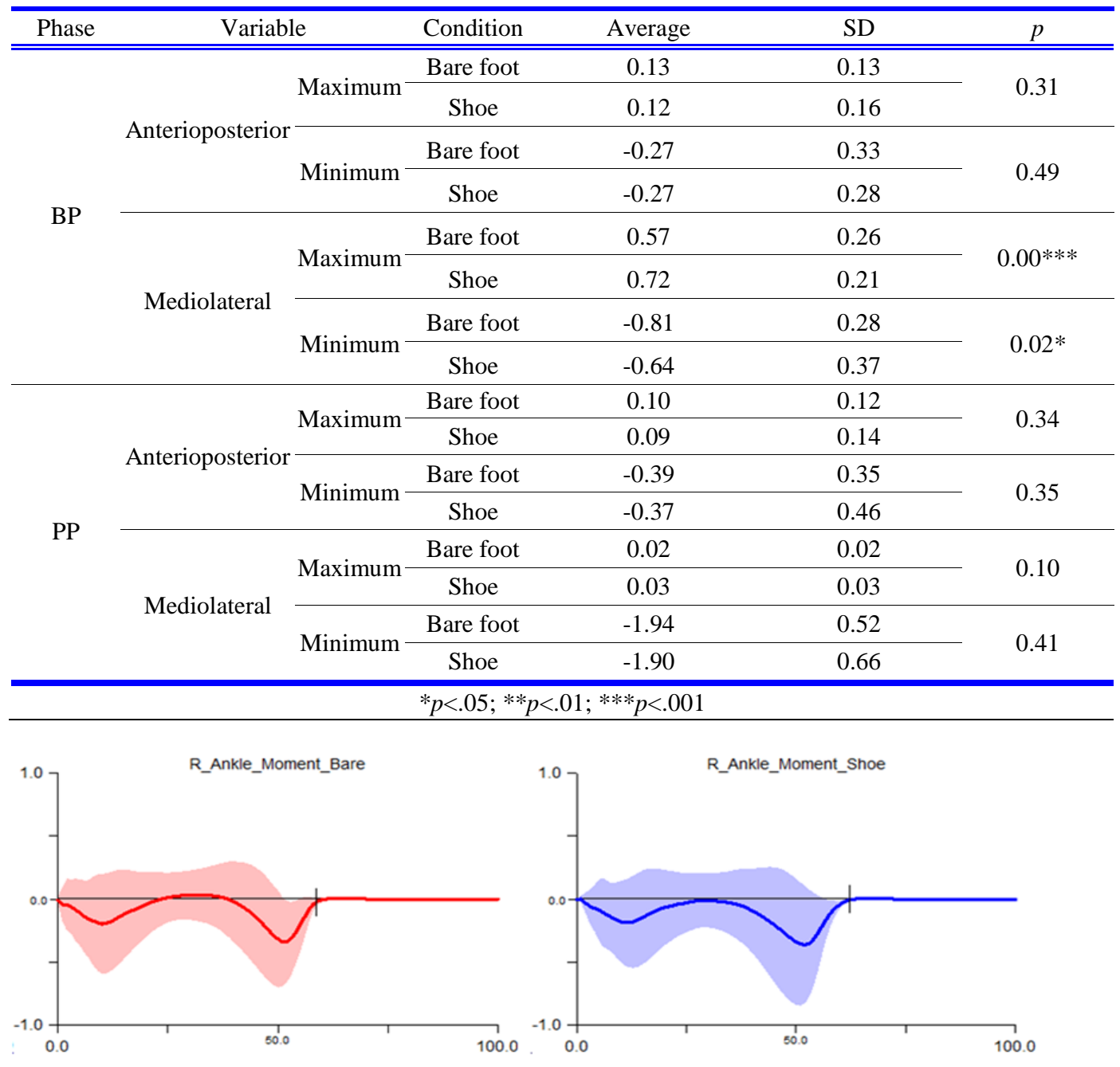




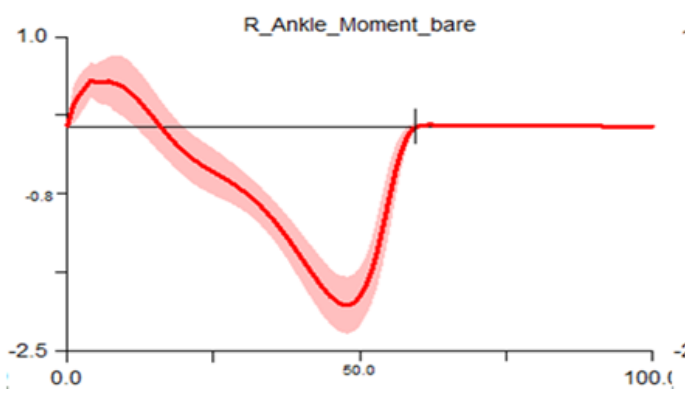

Figure 4. Ankle Moment of Bare

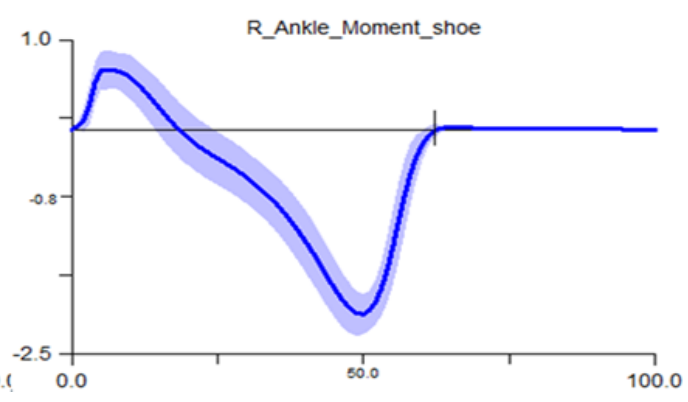

Figure 5. Ankle Moment of Shoe

\subsection{Knee Moments}

Knee moment about anterioposterior axis occurs during valgus and varus and knee moment about mediolateral axis occur during flexion and extension. No significant difference was observed except during BP phase (Table 4). This could be resulted from subjects utilizing knee movements more in order to compensate restricted ankle movement and to dissipate impact force. Even though these differences exist at impact phase, overall pattern didn't differ much during PP (Figure 6, 7).

Table 4. Knee Moment

\begin{tabular}{|c|c|c|c|c|c|c|}
\hline Phase & Variabl & & Condition & Average & SD & $p$ \\
\hline \multirow{8}{*}{$\mathrm{BP}$} & \multirow{4}{*}{ Anterioposterior } & \multirow{2}{*}{ Maximum } & Bare foot & 0.33 & 0.23 & \multirow{2}{*}{0.00} \\
\hline & & & Shoe & 0.17 & 0.14 & \\
\hline & & \multirow{2}{*}{ Minimum } & Bare foot & -1.07 & 0.45 & \multirow{2}{*}{0.08} \\
\hline & & & Shoe & -1.18 & 0.40 & \\
\hline & \multirow{4}{*}{ Mediolateral } & \multirow{2}{*}{ Maximum } & Bare foot & 1.69 & 0.48 & \multirow{2}{*}{0.02} \\
\hline & & & Shoe & 1.53 & 0.38 & \\
\hline & & \multirow{2}{*}{ Minimum } & Bare foot & -0.45 & 0.29 & \multirow{2}{*}{0.00} \\
\hline & & & Shoe & -0.70 & 0.27 & \\
\hline \multirow{8}{*}{ PP } & \multirow{4}{*}{ Anterioposterior } & \multirow{2}{*}{ Maximum } & Bare foot & 0.04 & 0.03 & \multirow{2}{*}{0.17} \\
\hline & & & Shoe & 0.03 & 0.04 & \\
\hline & & \multirow{2}{*}{ Minimum } & Bare foot & -0.87 & 0.55 & \multirow{2}{*}{0.16} \\
\hline & & & Shoe & -0.79 & 0.53 & \\
\hline & \multirow{4}{*}{ Mediolateral } & Moximum & Bare foot & 0.10 & 0.08 & \multirow{2}{*}{0.11} \\
\hline & & Maximum- & Shoe & 0.07 & 0.05 & \\
\hline & & \multirow{2}{*}{ Minimum } & Bare foot & -1.32 & 0.41 & \multirow{2}{*}{0.45} \\
\hline & & & Shoe & -1.30 & 0.49 & \\
\hline
\end{tabular}
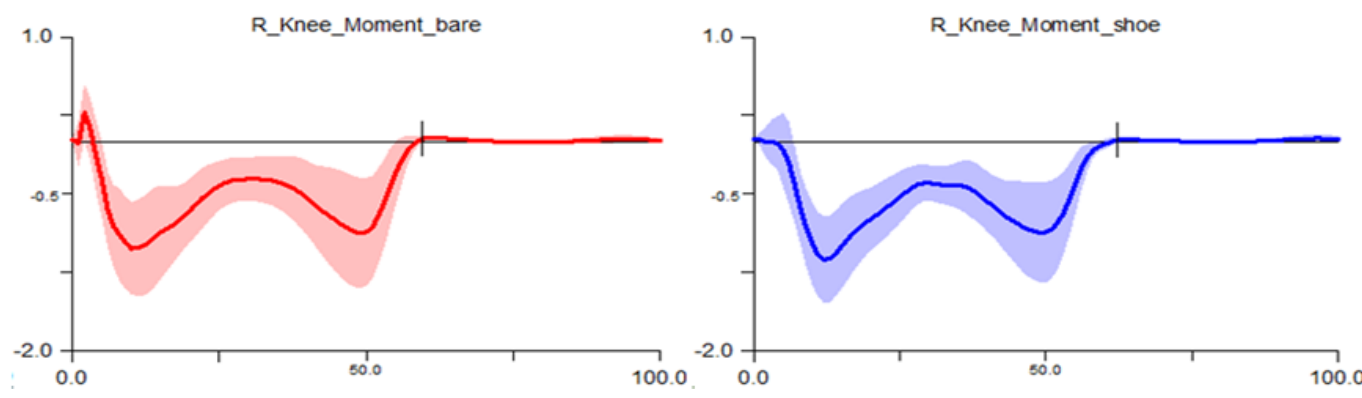


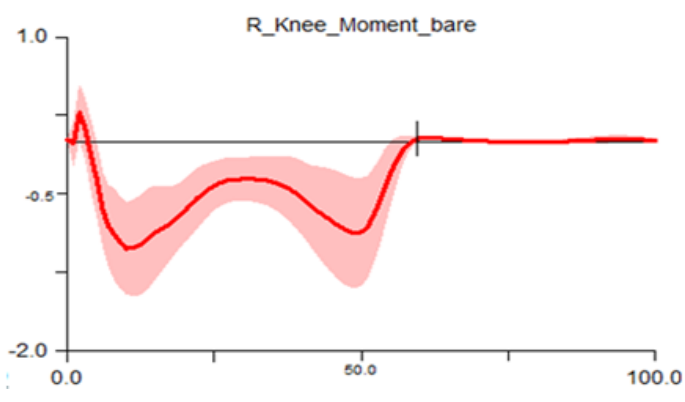

Figure 6. Knee Moment of Bare

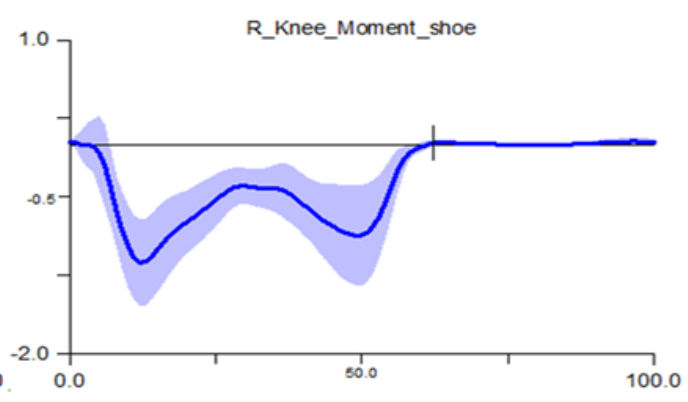

Figure 7. Knee Moment of Shoe

\subsection{Maximum Ground Reaction Force}

Ground reaction force is one of the important external force as well as gravity because human body uses ground reaction force to progress and control movements. Ground reaction force is often used to analyze biomechanical aspects of shoe's function during walking, running, and sprinting. Normally ground reaction force has two peaks during walking. First peak is where lower extremity extensors are eccentrically contacting to prevent body fro falling and second peak is where body's center of mass moves forward and propels body forward (Lee guong oak, 2006). The first peak is sometimes called maximum impact force or passive peak because it is resulted from a heel contacting the ground and brakes force passively. The second peak is sometimes called maximum propulsive force or active peak.

No significant difference was observed in peak vertical ground reaction force. However, peak anterioposterior and mediolateral ground reaction forces were significantly different during BP and peak anterioposterior ground reaction force during PP was significantly different (Table 5). This could be partially due to frictional force difference between bare foot and the shoes. Since the shoes exert greater frictional force to the ground than bare foot, during BP or braking phase subjects demonstrated greater values and bare foot with less frictional force had to exert greater push off force during PP or propulsive phase. It could be inferred with the similar manner that during BP phase bare foot had to go through more mediolateral movements due to less frictional force. Nevertheless, overall ground reaction force demonstrated similar pattern between the conditions (Figure 8, 9, and 10).

Table 5. Ground Reaction Force

\begin{tabular}{|c|c|c|c|c|c|}
\hline Phase & Variable & Condition & Average & SD & $p$ \\
\hline \multirow{6}{*}{$\mathrm{BP}$} & \multirow{2}{*}{ Vertical } & Bare foot & 834.36 & 99.94 & \multirow{2}{*}{0.39} \\
\hline & & Shoes & 837.80 & 96.98 & \\
\hline & \multirow{2}{*}{ Anterioposterior } & Bare foot & 15.77 & 31.80 & \multirow{2}{*}{$0.00^{* * *}$} \\
\hline & & Shoes & 47.22 & 31.79 & \\
\hline & \multirow{2}{*}{ Mediolateral } & Bare foot & 89.64 & 35.77 & \multirow{2}{*}{$0.02 *$} \\
\hline & & Shoes & 73.31 & 38.25 & \\
\hline \multirow{6}{*}{$\mathrm{PP}$} & \multirow{2}{*}{ Vertical } & Bare foot & 767.21 & 191.05 & \multirow{2}{*}{0.17} \\
\hline & & Shoes & 712.64 & 240.78 & \\
\hline & \multirow{2}{*}{ Anterioposterior } & Bare foot & 350.74 & 93.41 & \multirow{2}{*}{$0.04 *$} \\
\hline & & Shoes & 305.07 & 111.80 & \\
\hline & \multirow{2}{*}{ Mediolateral } & Bare foot & 4.08 & 5.43 & \multirow{2}{*}{0.07} \\
\hline & & Shoes & 6.83 & 9.53 & \\
\hline
\end{tabular}



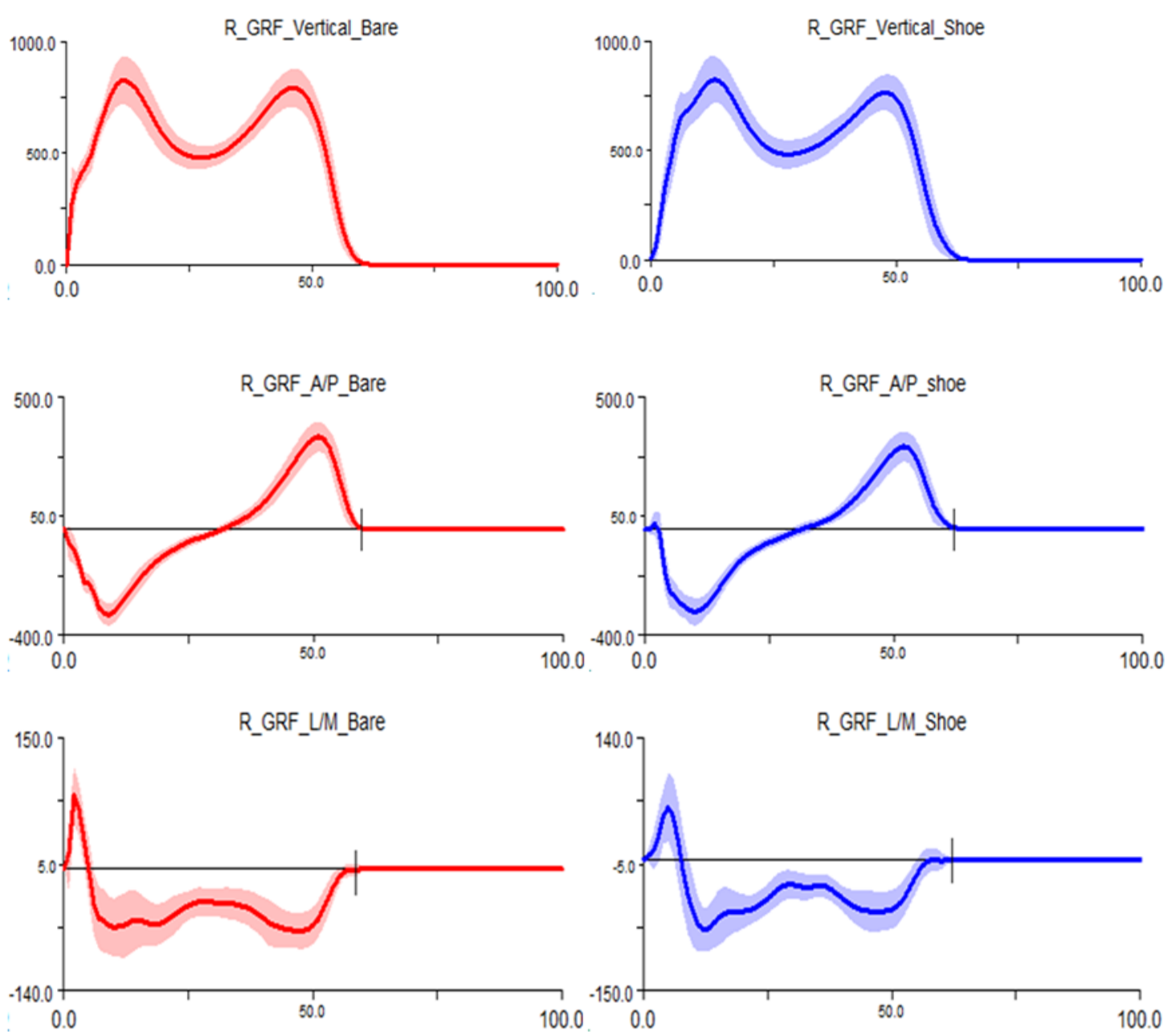

Figure 8. GRF of Bare

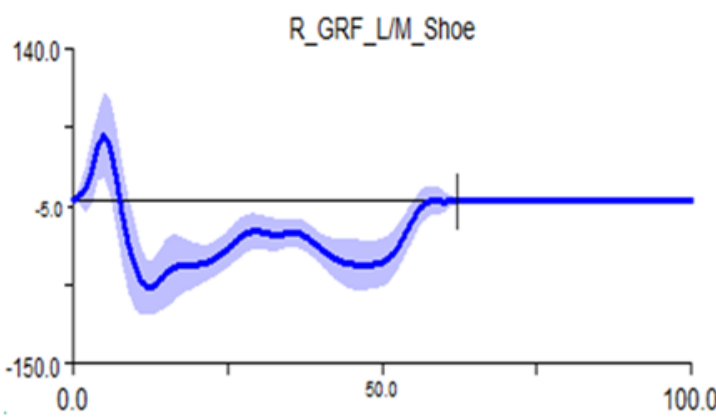

Figure 9. GRF of Shoe

\section{4. iEMG}

No significant difference was observed in all iEMG (Table 6). Slight increase in bare foot condition with Tibialis anterior could be due to the same reason as mentioned before. Subjects may have used impact force avoidance strategy and actively restricted their ankles

Table 6. iEMG

\begin{tabular}{|c|c|c|c|c|c|c|c|}
\hline \multirow{2}{*}{ Muscle } & \multirow{2}{*}{ Condition } & \multicolumn{3}{|c|}{ BP } & \multicolumn{3}{|c|}{ PP } \\
\hline & & Average & SD & $p$ & Average & SD & $p$ \\
\hline \multirow{2}{*}{ Rectus femoris } & Bare foot & 72.98 & 30.15 & \multirow{2}{*}{.124} & 20.31 & 10.27 & \multirow{2}{*}{.106} \\
\hline & Shoe & 79.19 & 48.62 & & 26.13 & 18.64 & \\
\hline \multirow{2}{*}{ Biceps femoris } & Bare foot & 89.74 & 45.72 & \multirow{2}{*}{.272} & 15.26 & 9.81 & \multirow{2}{*}{.234} \\
\hline & Shoe & 90.18 & 43.10 & & 22.13 & 8.89 & \\
\hline \multirow{2}{*}{ Gastrocnemius } & Bare foot & 152.84 & 52.61 & \multirow{2}{*}{$.02 *$} & 75.16 & 15.43 & \multirow{2}{*}{.167} \\
\hline & Shoe & 188.39 & 67.04 & & 79.67 & 16.89 & \\
\hline \multirow{2}{*}{ Tibialis anterior } & Bare foot & 126.47 & 57.93 & \multirow{2}{*}{.097} & 223.84 & 91.28 & \multirow{2}{*}{.068} \\
\hline & Shoe & 120.66 & 88.23 & & 201.58 & 102.94 & \\
\hline
\end{tabular}




\section{Conclusion}

This study examined the effects of functional shoes which is being developed to have similar effects of bare foot walking through subject test with quantitative analysis. Loading patterns during walking revealed with joint moments and ground reaction force are as follows;

(1) Joint moments were similar during most of phases. However, less ankle moment and greater knee moment were observed during BP phase.

(2) Similar pattern was observed with ground reaction force data. However, greater magnitude in anteriopoestrior component during BP phase in function shoe condition and greater magnitude during PP phase in bare foot condition.

(3) No significant difference was observed with iEMG magnitude in major lower extremity muscles between the conditions

\section{Acknowledgements}

This study was supported by the Research Program funded by the Seoul National University of Science and Technology (2015- ) $)$.

\section{References}

[1] P. R. Cavanagh, G. C. Andrew, R. Kram, M. M. Rodgers, D. J. Sanderson, and E. M. Hennig, "An approach to biomechanical profiling of elite distance runners", Int. Journal of Sports biomechanics, vol. 1 , no. 1 , (1985), pp. 36-62.

[2] G. L. David and F. B. Thor, "An EMG-driven musculoskeletal model to estimate muscle forces and knee joint moments in vivo", Journal of Biomechanics, vol. 36, no. 6, (2003), pp. 765-776.

[3] A. L. Hof, H. Elzinga and W. Grimmius, "Speed Dependence of averaged EMG profiles in walking", Gait and Posture, vol. 16, no. 1, (2003), pp. 78-86.

[4] R. Kerr, G. Arnold, L. Cochrane, T. Drew and R. Abboud, "The effect of shoes on ankle injuries", Journal of Biomechanics, vol. 39, (2006), p. 33.

[5] D. C. Kerrigan, J. L. Lelas and M. E. Karvosky, "Women's shoes and knee osteoarthritis", The Lancet, vol. 357, (2001), p. 1097

[6] W. Kim and A. S. Voloshin, "Role of plantar fascia in the load bearing capacity of the human foot", Journal of Biomechanics, vol. 28, no. 9, (1995), pp. 1025-1033.

[7] R. A. Mann, "Biomechanics of running", Symposium on the Leg, Running Sports, R. P., Mack (Ed.) St. Louis: The C.V. Mosby Co., (1980), pp. 1-29.

[8] J. C. Menant, S. D. Perry, J. R. Steele, H. B. Menz, B. J. Munro and S. R. Lord, "Effects of shoe characteristics on dynamic stability when walking on even and uneven surfaces in young and older people", Arch Phys Med Rehabil., (2008) 89, 1970.

[9] W. W. Michael, "Gait analysis: An Introduction, Oxford Orthopaedic Engineering Centre, University of Oxford”, (1994), pp. 54-74.

[10] B. M. Nigg and A. H. Bahlsen, "Factors influencing kinematic variables in running", In Biomechanics of Running Shoes, M. M. Nigg(ED). Champaign, III: Human Kinetics Publishers Inc., (1986), pp. 139-159.

[11] B. M. Nigg and W. Liu, "The elect of muscle stiffness and damping on simulated impact force peaks during running", Journal of Biomechanics, vol. 32, (1999), pp. 849-856.

[12] B. B. Nigg, "The role of impact forces and foot pronation: a new paradigm", Clinical Journal of Sport Medicine, vol. 11, no. 1, (2001), pp. 2-9.

[13] B. Nigg, S. Hintzen and R. Ferber, "Effect of an unstable shoe construction on lower extremity gait characteristics", Clinical Biomechanics, vol. 21, (2006), pp. 82-88.

[14] D. Oeffinger, B. Brauch, S. Cranfill, C. Hisle, C. Wynn, R. Hicks and S. Augsburger, "Comparison of gait with and without shoes in children", Gait and Posture, vol. 9, (1999), pp. 95-100.

[15] R. M. Queen, A. N. Abbey, J. I. Wiegerinck, J. C. Yoder and A. N. James, "Effect of shoe type on plantar pressure: A gender comparison", Gait and Posture, vol. 31, (2010), pp. 18-22.

[16] E. Sobel, S. J. Levitz and M. A. Caselli, "Orthoses in the treatment of rearfoot problems", Journal of American Podiatry Medicine Association, vol. 89, no. 5, (1999), pp. 220-233.

[17] J. Tomaro and R. G. Burdett, "The effects of foor orthotics on the EMG activity of selected leg muscles during gait", The Journal of Orthopacedic and Sports Physical Therapy, vol. 18, no. 4, (1993), pp. 532536. 


\section{Author}

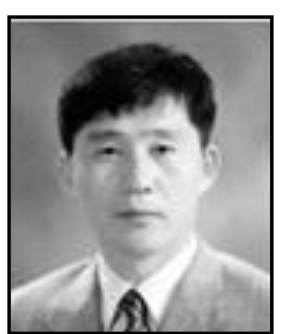

\section{Chong-hoon Lee}

Dept. of Sports Science,

Seoul National University of Science \& Technology

138 Gongreung-gil, Gongreung2-dong 172, 139-743, Korea

Email: leejh36@snut.ac.kr 
International Journal of Bio-Science and Bio-Technology

Vol.7, No.3 (2015) 